\title{
Aspectos morfológicos e comportamentais dos machos de Ancyloscelis Latreille (Anthophoridae, Apoidea)
}

\author{
Isabel Alves-dos-Santos ${ }^{1}$
}

\begin{abstract}
Morphological and behavioral aspects of Ancyloscelis Latreille males (Anthophoridae, Apoidea). Specimens of five bee species of the genus Ancyloscelis Latreile, 1836 were sampled during three consecutive years in Rio Grande do Sul, Brazil, with the aim to study their morphology and behavior during flower visits. Characteristics that allow to distinguish the five species are presented.

KEY WORDS. Hymenoptera, solitary bees, legs morphology, patrol behavior
\end{abstract}

O gênero Ancyloscelis Latreile, 1836 foi referido pela primeira vez por LATREILLE (1825), porém sem incluir nenhuma descrição. O gênero foi considerado nomen nudum por ter sido proposto e descrito desacompanhado da designação do genótipo (MicHENER 1942). A primeira espécie incluída foi $A$. ursinus, designada espécie tipo do gênero (HALIDAY 1836). MourE (1992) e RoIG-ALSINA \& MiCHENER (1993) retiraram Ancyloscelis da subfamília Exomalopsinae. J.S. Moure (comunicação pessoal) considera o gênero pertencente à tribo Melitomini por possuir pilosidade que acompanha a gálea típica para o grupo e a dilatação das pernas posteriores nos machos. RoIG-ALSINA \& MICHENER (1993) desmembraram a tribo Exomalopsini e o gênero Ancyloscelis foi agrupado aos Emphorini em uma nova subtribo Ancyloscelina, que também estaria muito próxima de gêneros tais como Melitoma e Ptilothrix da outra subtribo Emphorina.

O gênero Ancyloscelis é composto por abelhas de tamanho pequeno a moderado, com corpo negro e clípeo fortemente protuberante. As espécies são amplamente distribuídas na região Neotropical, ocorrendo desde a Argentina até sudoeste dos E.U.A.. Existe muito mais diversidade de estruturas entre as espécies sul americanas que entre aquelas da América Central e do Norte (MichenER \& MOURE 1957).

A morfologia e função da probóscide das fềmeas de Ancyloscelis, ocorrentes no Rio Grande do Sul, foram estudadas, bem como o papel destas abelhas como polinizadoras (ALVES-DOS-SANTOS 1997; SCHLINDWEIN \& WITTMANN 1995). No presente trabalho foram reconhecidas as cinco espécies de Ancyloscelis que ocorrem no Rio Grande do Sul, especial caracterização é dada aos machos e seus comportamentos de patrulha nas flores.

1) Laboratório de Abelhas, Departamento de Ecologia Geral, Instituto de Biociências, Universidade de São Paulo. Cidade Universitária, 05508-900 São Paulo, São Paulo, Brasil. E-mail: isabelha@usp.br 


\section{MATERIAL E MÉTODOS}

Exemplares de Ancyloscelis, machos e fêmeas, foram coletados em flores ao longo de três anos consecutivos (1991-1994), principalmente em áreas dos Municípios de Guaíba, Viamão e Terra de Areia, todos localizados na Planície Costeira do Rio Grande do Sul. A identificação das abelhas foi feita com auxílio do Prof. Dr. J.S. Moure. Foram feitas observações no campo, sobre suas visitas às flores e o comportamento de patrulha dos machos. Fotos de microscopia eletrônica de varredura foram realizadas na Universidade de Tübingen com auxílio do Prof. Dr. Dieter Wittmann. O material examinado encontra-se depositado na coleção de referência do Laboratório de Pesquisas Biológicas da Universidade de Tübingen na Pontifícia Universidade Católica do Rio Grande do Sul (PUCRS), Porto Alegre.

\section{RESULTADOS E DISCUSSÃO}

No Rio Grande do Sul ocorrem cinco espécies de Ancyloscelis: A. apiformis (Fabricius, 1793), A. fiebrigi Brèthes 1909, A. gigas Friese 1904, A. turmalis Vachal 1904 e A. ursinus Haliday 1836. As abelhas deste gênero possuem longa probóscide, que pode apresentar densa pilosidade (A. gigas, A. ursinus e A.turmalis) ou ser praticamente glabra (A. apiformis $\mathrm{e}$. fiebrigi). Os machos de Ancyloscelis possuem as pernas posteriores extremamente dilatadas (coxa, fêmur e tíbia) mostrando um acentuado dimorfismo sexual (Fig. 1). O basitarso do terceiro par de pernas, modificado em forma de "gancho", é típico para reconhecer as espécies. A tíbia posterior muitas vezes apresenta uma protuberância, como um dente truncado (ausente em A. apiformis). Os dados apresentados na tabela I e figuras 2 e 3 auxiliam na identificação das espécies.

Tabela I. Caracteres dos machos das espécies de Ancyloscelis ocorrentes no Rio Grande do Sul.

\begin{tabular}{lcll}
\hline Espécies & $\begin{array}{c}\text { Comprimento total } \\
\text { aproximado }(\mathrm{mm})\end{array}$ & \multicolumn{1}{c}{$\begin{array}{c}\text { Forma do } \\
\text { basitarso posterior }\end{array}$} \\
\hline $\begin{array}{l}\text { A. apiformis } \\
\text { A. fiebrigi }\end{array}$ & 62 & $\begin{array}{c}\text { Com cavidade profunda e espinho forte } \\
\text { Com cavidade mais alongada do que profunda, } \\
\text { espinho fraco }\end{array}$ & $\begin{array}{c}\text { Tubérculo na } \\
\text { coxa média }\end{array}$ \\
$\begin{array}{l}\text { A. gigas } \\
\text { A. turmalis }\end{array}$ & 82 & Com cavidade espinho & Forte e truncado \\
A. ursinus & 803 & Com cavidade e espinho & Proeminente \\
\hline
\end{tabular}

O basitarso é típico para cada espécie. Apenas em A. ursinus o basitarso é liso (Figs 2E, 3E-F). Nas outras quatro espécies o basitarso possui uma cavidade e espinho. Em $A$. apiformis a cavidade é pronunciada e o espinho forte (Figs 2A, $3 \mathrm{~A}-\mathrm{B}$ ), enquanto que em $A$. fiebrigi a cavidade é mais alongada do que profunda e o espinho fraco (Fig. 2B). O basitarso destas espécies de Ancyloscelis lembra na sua forma o gancho (Klammerhaftapparat) de Apis florea descrito por RUTTNER (1975), presente nas pernas posteriores dos machos e com função de segurar a fêmea. Segundo TORO (1985) caracteres presentes só nos machos, parecem estar relacionados fundamentalmente ao mecanismo de cópula. Esta estrutura nos machos de Ancyloscelis parece exercer função semelhante àquela esquematizada por RUTTNER 


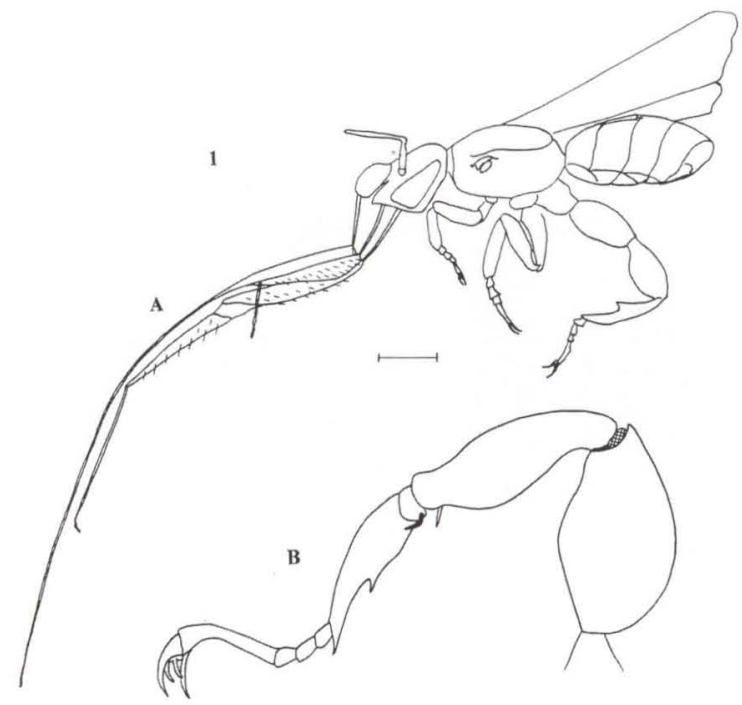

Fig. 1. (A) Macho de Ancyloscelis gigas, esquema (Escala= $1 \mathrm{~mm}) ;(B)$ perna posterior do macho de $A$. gigas, esquema.
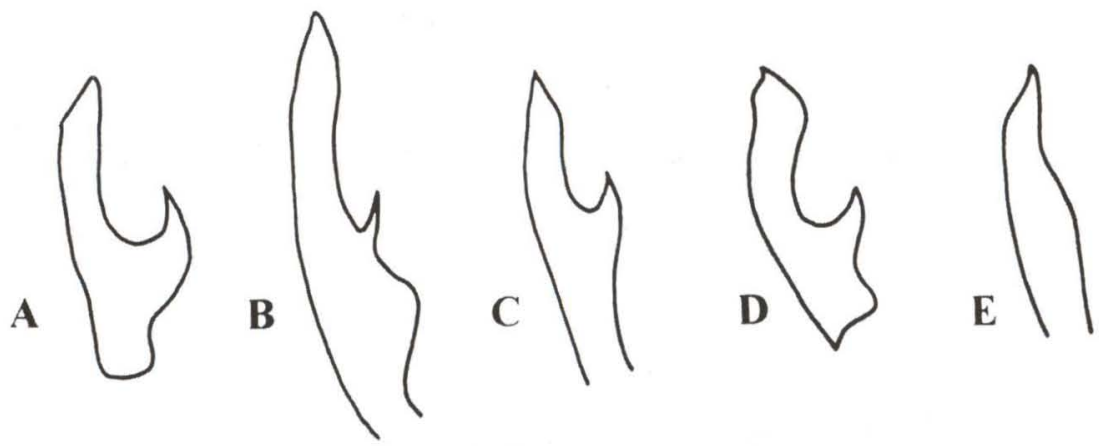

2

Fig. 2. Basitarsos das pernas posteriores dos machos de Ancyloscelis. (A) A. apiformis; (B) A. fiebrigi; (C) A. gigas; (D) A. turmalis; (E) A. ursinus.

(1975) durante a cópula em A. florea, promovendo provavelmente, um bom encaixe com as tíbias das pernas posteriores da fêmea, evidenciando assim vantagens mecânicas para uma cópula de longa duração.

Quanto à coloração, os machos de $A$. apiformis e $A$. fiebrigi são pretos, com pernas marrom-avermelhadas, clípeo com faixa amarelo-clara, labro e base da mandíbula amarelo-claros. Em A. gigas os machos são pretos, com pernas negras, pilosidade dos últimos tergos amarela clara; labro e base da mandíbula amarelo-claros, clípeo com pontuação muito grossa e faixa amarela mais escura. Em A. turmalis e $A$. ursinus os machos são marrom-amarelados ou dourados, com pernas pretas ou marrons com pilosidade dourada e tarsos amarelos; clípeo com faixa larga amarela escura, labro e base da mandíbula amarelo-escuros. 

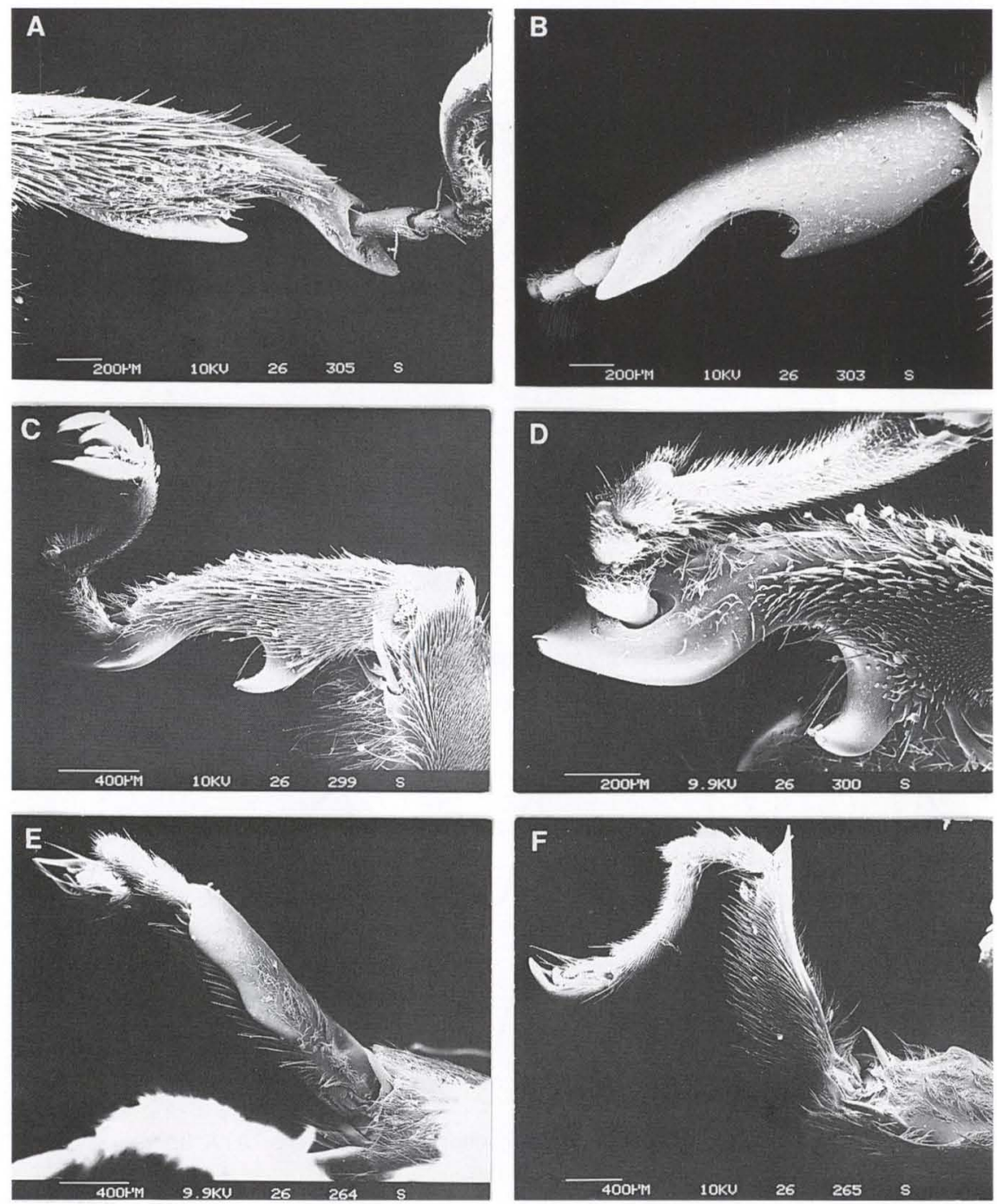

Fig. 3. Microscopia eletrônica de varredura dos basitarsos dos machos de Ancyloscelis. (A, B) A. apiformis, face externa e interna; (C, D) A. turmalis, face interna; $(E, F) A$. ursinus, face externa e interna.

As espécies de Ancyloscelis apresentam uma clara preferência floral, e algumas delas são altamente especializadas em suas fontes polínicas. Estas abelhas são frequentemente encontradas em flores de Pontederiaceae (A. gigas, $A$. ursinus e A. turmalis), Convolvulaceae (A. apiformis e A. fiebrigi) e Cactaceae (A. fiebrigi). Machos de Ancyloscelis, de todas as cinco espécies, foram observados patrulhando ostensivamente e/ou esperando as fêmeas junto às respectivas plantas. Eles circulam entre as flores ou inflorescências fazendo na maioria das vezes um circuito em "8", 

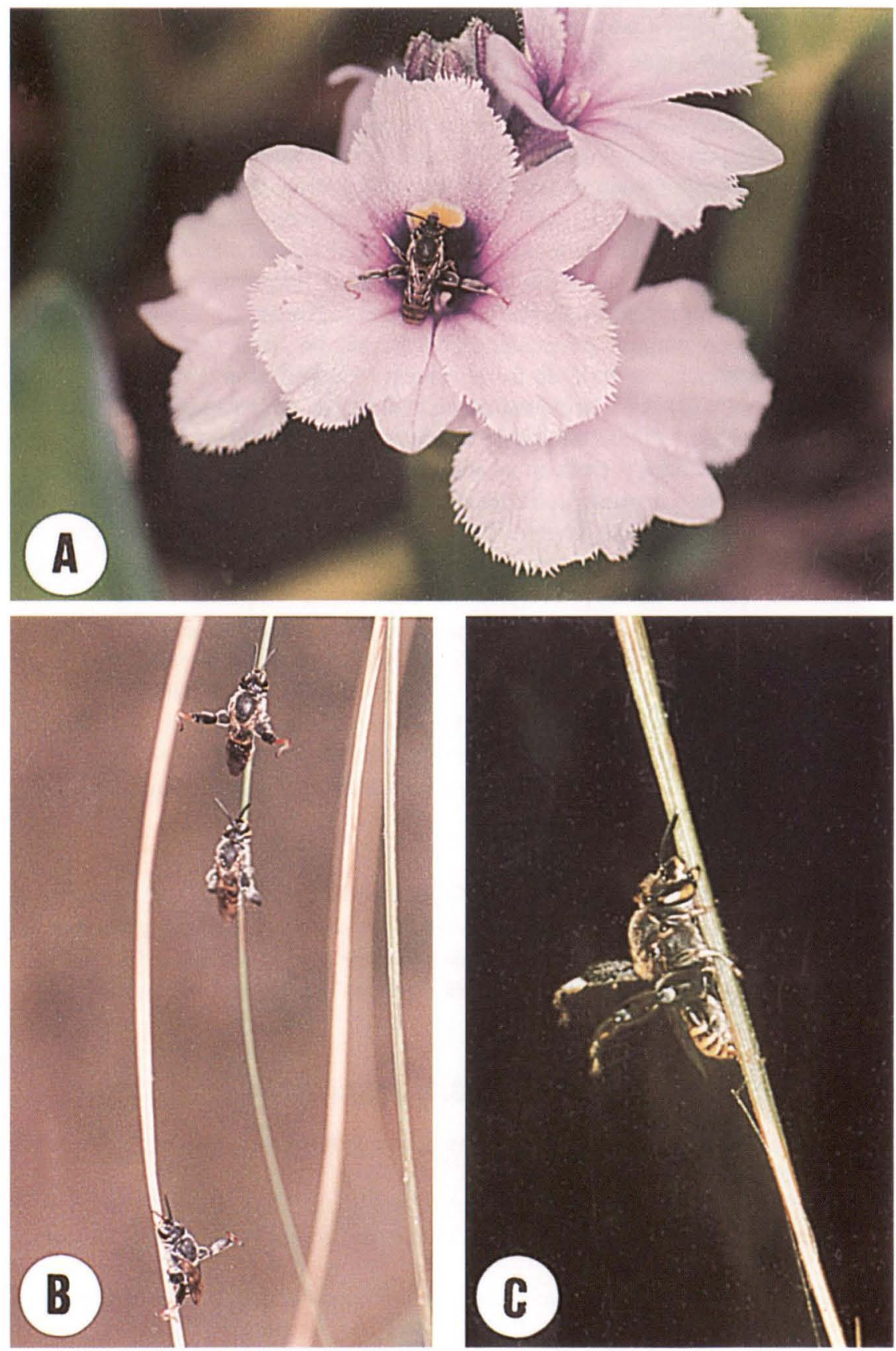

Fig. 4. (A) Macho de Ancyloscelis gigas pousado sobre a flor de Eichhornia azurea; $(B, C)$ machos de $A$. gigas no local de dormir. 
depois pousam sobre a folhagem ou sobre uma flor, cobrindo a entrada da mesma (Fig. 4A). Quando estão pousados na folhagem, permanecem de prontidão patruIhando a inflorescência, a cerca de $10-15 \mathrm{~cm}$ de distância. Ao menor movimento ou aproximação de um inseto, voam rapidamente para averiguar a presença da fêmea. Não se tratando da mesma, geralmente voltam para o mesmo local na folhagem. Este comportamento é mais frequente no período da tarde. Durante estes pousos os machos esporadicamente estendem a probóscide, aparentando uma desidratação de néctar. No periodo da manhã, a patrulha sobre as inflorescências é feita com raras interrupções. No período da tarde pousam com mais frequência. A cópula geralmente ocorre na flor visitada pela fêmea. O número de fêmeas é, proporcionalmente, muito inferior ao número de machos patrulhando no local (cerca de 1/15). A ocorrência da cópula no local de forrageamento é relativamente comum entre as abelhas (ALCOCK 1984), e principalmente fácil de ser observada em abelhas que possuem fontes alimentícias altamente especializadas. Em casos como estes, é bem provável que os machos patrulhem na área de ocorrência das plantas. Os machos de Ancyloscelis visitam as mesmas flores das fêmeas para tomar néctar. O patrulhamento ostensivo significa um enorme gasto energético para estes machos. A diminuição das atividades no período da tarde pode ser, entre outros fatores, resultante da diminuição da produção e oferta de néctar nas plantas.

Além de patrulhar na área de forrageamento da fềmea, foi observado que os machos possuem um local de dormir (sleepingplace) próximo às plantas visitadas (observado para A. gigas, A. turmalis e A. ursinus). Os machos geralmente agrupam-se no final da tarde, agarram-se a filetes da vegetação com as mandíbulas e estendem as pernas posteriores (Fig. 4B-C), explicando assim a razão pela qual MiCHENER (1974) e TORCHIO (1974) não encontraram machos próximos aos ninhos de Ancyloscelis armata na Colômbia.

AGRADECIMENTOS. Agradeço ao Prof. Dieter Wittmann pela orientação, colaboração, apoio e valiosas sugestões neste trabalho, ao Prof. J.S. Moure pela constante colaboração e principalmente pela minha iniciação no mundo das abelhas, e aos biólogos Alice Grossman, Mardiore Pinheiro, Rodrigo Cunha e Paulo Milani pelas valiosas contribuições nos trabalhos de campo e laboratório.

\section{REFERÊNCIAS BIBLIOGRÁFICAS}

AlCoCK, J. 1984. Animal Behavior, an Evolutionary Approach. Sunderland, Sinauer Associates Inc. Publishers, $5^{\text {th }}$ ed., 625p.

Alves-DOS-SAntos, I. 1997. Melittophilous Plants, Their Pollen and Flower Visiting Bees in Southern Brazil: 3. Pontederiaceae. Biociências, Porto Alegre, 5 (2): 3-18.

HALIDAY, A.H. 1836. Descriptions of Hymenoptera collected by Capt. King in the survey of Straits of Magellan. Trans. Linn. Soc. London 17: 316-331.

LAtreille, P.A. 1825. Families naturelles du règne animal. Paris, Baillière, 570 p.

MichenER, C.D. 1942. North American Bees of the Genus Ancyloscelis 
(Hymenoptera: Anthophoridae). Pan-Pacific Entomol. 18 (3): 108-113.

. 1974. Further notes on nests of Ancyloscelis (Hymenoptera: Anthophoridae). Jour. Kansas Entomol. Soc. 42 (1): 19-22.

MichenER, C.D. \& J.S. MouRE. 1957. A study of the classification on the more primitive non-parasitic anthophorine bees (Hymenoptera, Apoidea). Amer. Mus. Nat. Hist. 112 (5): 399-451.

MourE, J.S. 1992. Lissopedia, gen.n. de Paratetrapedini para região Neotropical, com as descrições de três espécies novas (Hymenoptera, Apoidea, Anthophoridae). Revta bras. Zool. 9 (3-4): 305-317.

RoIG-AlsinA, A. \& C.D. MiChEnER. 1993. Studies of the phylogeny and classification of long-tongued bees (Hymenoptera:Apoidea). Univ. Kansas Sci. Bull. 55 (4): 123-173.

RUTTNER, F. 1975. Ein metatarsaler Haftapparat bei den Drohnen der Gattung Apis (Hymenoptera: Apidae). Entomol. Germanica 2 (1): 22-29.

SCHLINDWEIN, C. \& D. WitTMANN. 1995. Specialized solitary bees as effective pollinators of South Brazilian species of Notocactus and Gymnocalycium (Cactaceae). Bradleya 13: 25-34.

TORCHIO, P.F. 1974. Notes on the biology of Ancyloscelis armata Smith and comparisons with other anthophorine bees. Jour. Kansas Entomol. Soc. 47 (1): 54-63.

Toro, H. 1985. Ajuste mecanico para la copula de Callonychium chilense (Hymenoptera, Andrenidae). Rev. Chil. Ent. 12: 153-158.

Recebido em 06.VIII.1998; aceito em 16.XI.1999. 